\title{
Quantitative imaging of haematopoietic stem and progenitor cell localization and hypoxic status in the bone marrow microenvironment
}

\author{
César Nombela-Arrieta, Gregory Pivarnik, Beatrice Winkel, Kimberly J. Canty, Brendan Harley, John E. \\ Mahoney, Shin-Young Park, Jiayun Lu, Alexei Protopopov and Leslie E. Silberstein
}

Nat. Cell Biol. 15, 533-543 (2013); published online 28 April 2013; corrected after print 26 June 2013

In the version of this Technical Report originally published, reference to a study (L. Wang et al. Identification of a clonally expanding haematopoietic compartment in bone marrow. EMBO J. 32, 219-230; 2013) that used confocal microscopy to analyse the presence of phenotypically defined HSPCs in previously unreported and localized structures termed hemospheres, published while the current manuscript was under consideration, was inadvertently omitted. This has now been added as reference 42 , and the following paragraph has been added to the Discussion section.

"While this publication was under revision, a study was published that described the existence of hemospheres, small and localized bone mar-
row regions enclosed within bone trabeculae in distal portions near the growth plate of femoral bones ${ }^{42}$. Hemospheres contain bone marrow
microvessels, stromal cells of mesenchymal origin, haematopoietic cells and a small but significant fraction of perivascular HSPCs. Of note,
HSPCs within hemospheres were exclusively found to rapidly expand in a clonal fashion during homeostasis and following transplantation
post-irradiation ${ }^{42}$. It is therefore conceivable that hemospheres represent specific perivascular niches, with different instructive properties to
those found in other perivascular HSPC microenvironments throughout the bone marrow cavity. Further defining the distinctive cellular and
molecular features of hemospheres will be critical to ultimately determine the functional role of these previously unrecognized compartments."

Furthermore, in the original version of this Technical Report, the e-mail address for the co-corresponding author César Nombela-Arrieta was incorrect, it should have read: Cesar.NombelaArrieta@childrens.harvard.edu.

These errors have now been corrected in the HTML and PDF versions of the Technical Report.

\section{TFEB controls cellular lipid metabolism through a starvation- induced autoregulatory loop}

Carmine Settembre, Rossella De Cegli, Gelsomina Mansueto, Pradip K. Saha, Francesco Vetrini, Orane Visvikis, Tuong Huynh, Annamaria Carissimo, Donna Palmer, Tiemo Jürgen Klisch, Amanda C. Wollenberg, Diego Di Bernardo, Lawrence Chan, Javier E. Irazoqui and Andrea Ballabio

Nat. Cell Biol. 15, 647-658 (2013); published online 21 April 2013; corrected online 25 April 2013

In the version of this Article originally published, in the Methods section, Supplementary Table S8 was incorrectly cited as listing lipid metabolism gene-specific primers under the heading 'RNA extraction, quantitative PCR and statistical analysis'. The list of gene-specific primers has now been uploaded as Supplementary Table S10 and the citation in the Methods has been amended. This has been corrected in the PDF and HTML versions of this Article. 